tief angelegten medienhistoriographischen $\mathrm{Be}-$ trachtung des deutschen Sprach- und Kulturraumes statt der Zeit der Weimarer Republik nicht doch die des Dritten Reiches mit seiner bemerkenswerten medientechnologischen, wenn auch umstrittenen medienpolitischen Entwicklung, die End- und zugleich paradoxer Wendepunkt vieler Modernisierungsprozesse auch im Medialen war, hätte setzen sollen, ließe sich sicherlich diskutieren. Der Autor hat jedoch sowohl die Zeiten der Nazi-Diktatur als auch die der Bundesrepublik Deutschland medienhistorisch in anderen Werke erfasst und es ist sicherlich sein gutes Recht, entsprechende Periodisierungen vorzunehmen.

Es sei hervorgehoben, dass ähnliche wertvolle medienhistorische Gesamtdarstellungen über die verschiedenen Zeiten und Epochen hinweg nur für wenige europäische Länder vorliegen, aber unbedingt notwendig sind. Denn, wie der Autor selbst im Nachwort schreibt: „Wenn heute vom Wandel zur Informationsgesellschaft die Rede ist, dann mag der Blick zurück zeigen, wie weit der Weg bis dahin gewesen ist und welche Beschleunigung dabei stattgefunden hat".

Da die heutige gesellschaftliche Entwicklung nicht nur unter dem Vorzeichen der Informationsgesellschaft, sondern auch dem der Globalisierung und des erweiterten europäischen Einigungsprozesses verläuft, könnten solche umfassenden Darstellungen der Medienentwicklung in einem Land oder in einem sprachkulturellen Raum auch eine gute Grundlage bilden für eine in Zusammenarbeit von Medienhistorikern aus allen Teilen des alten Kontinents erstellte Medien- und Kommunikationsgeschichte Europas.

\section{Rossen Milev}

\section{Christina Holtz-Bacha}

\section{Wahlwerbung als politische Kultur}

Parteienspots im Fernsehen 1957 - 1998

Wiesbaden: Westdeutscher Verlag,

2000. $-270 \mathrm{~S}$.

\section{ISBN 3-531-13551-1}

Eine gewisse Wahlmüdigkeit kann nicht nur der deutschen Wählerschaft, sondern auch den deutschen Wahlforschern attestiert werden. Nach Schweigespirale, Agenda-Setting, Personalisierung, Amerikanisierung und Theatralisierung fehlt gegenwärtig ein zündendes Konzept, mit dem die Forschenden zu neuen Kon- troversen gereizt werden könnten. Der tendenzielle Stillstand auf der Theorieebene lenkt die Aufmerksamkeit auf konkrete wahlkämpferische Forschungsobjekte wie etwa den Parteienspot im Fernsehen, der bislang von den arrivierten deutschen Politik- und Kommunikationswissenschaftlern weitgehend ignoriert wurde.

Die Autorin, Professorin für Publizistik an der Universität Mainz, langjährige Sprecherin der DGPuK-Fachgruppe Politik und Kommunikation sowie Mitherausgeberin der Fachzeitschrift „Publizistik“, legt mit ihrer Monografie eine Langzeitstudie der Wahlwerbespots in zwölf Bundestagswahlkämpfen seit 1957 vor.

Das Buch ist in fünf Kapitel gegliedert und mit zahlreichen Tabellen und Schaubildern, jedoch keinem Register versehen. Im ersten $\mathrm{Ka}$ pitel wird Wahlwerbung als eine „Kommunikationsform mit Doppelfunktion" definiert, die kurzfristig ein persuasives Kommunikationsziel verfolgt und langfristig Teil von politisch-kulturellen Deutungsmustern wird. Im zweiten Kapitel folgt ein Exkurs zum „Vorbild USA“. Hier wird die amerikanische Forschungsgeschichte sowie der Forschungsstand zum Wahlspot unter besonderer Berücksichtigung der wichtigen Arbeiten von Kathleen Hall Jamieson, Edwin Diamond/Stephen Bates sowie Lynda Lee Kaid rezipiert, jedoch ohne die deutschsprachige Rezeption amerikanischer Wahlkampfkommunikation zu berücksichtigen. Kapitel drei fasst die Vorgeschichte sowie den Forschungsstand der Wahlwerbung und ihrer Rezeption in der Bundesrepublik zusammen, während Kapitel vier eine "Chronik der Bundestagswahlkämpfe“ bietet, wobei Christina Holtz-Bacha die vierzig untersuchten Jahre in fünf Perioden unterteilt: Wahlkämpfe im Nachkriegsdeutschland, Die Phase der Konsolidierung, Aufbruch und Polarisierung, Hoffnung und Zuversicht sowie Wahlen im vereinten Deutschland. In den jeweiligen Unterkapiteln sind die einzelnen Wahlkämpfe auf wenigen Seiten zusammengefasst, so dass sich dieses Kapitel als handliches Nachschlagewerk zu Themen, Kommunikation und Kontext der Bundestagswahlkämpfe eignet.

Der empirische Kern der Langzeitstudie wird abschließend in Kapitel fünf präsentiert: „Wahlspots im deutschen Fernsehen 1957 1998“. Dabei unterscheidet sich das gewählte Forschungsdesign von vergleichbaren USamerikanischen Untersuchungen. Deutsche 
Vergleichsstudien lagen bislang nicht vor. Die Autorin und ihr Forschungsteam konzentrierten sich nicht auf die gesamten Spots als Untersuchungskategorie, sondern auf die einzelnen Sequenzen. Die Analyseeinheit ist dabei kleiner und bezieht sich auf eine Sequenz/Szene, die besteht „aus einer oder mehreren Einstellungen, die durch Schnitt oder Überblendungen voneinander getrennt sind" (S. 151). Konsequent findet sich im Anhang eine Übersicht über sämtliche Sequenzen aller Parteien seit 1957. Die SPD ist mit 631 Sequenzen deutlicher Spitzenreiter vor der CDU mit 483 Sequenzen, jedoch nur, da die CSU mit 156 Sequenzen extra gezählt wurde. Die FDP rangiert mit 182 Sequenzen auf Platz drei. Die Spots von Bündnis 90/Die Grünen summieren sich immerhin auf 78 Sequenzen. Die NPD ist mit 40 Sequenzen unter den außerparlamentarischen Parteien diejenige mit der höchsten Sequenzzahl. Dieses Bild spiegelt sich auch bei der Verteilung der Spots auf die Parteien wider (S. 155): Von insgesamt 417 untersuchten Spots entfielen über den gesamten Untersuchungszeitraum auf die SPD 104, auf die CDU 101 und auf die CSU 32. Die FDP beteiligte sich zwischen 1957 und 1998 mit insgesamt 39 Spots, Bündnis 90/Die Grünen mit 20 Spots an der TV-Wahlwerbung. Die Datenfülle beeindruckt, zumal ihr eine mühselige und akribische Quellenrecherche vorausgegangen sein muss. Denn TV-Spots gehören zu den ephemeren Kommunikationsmitteln, die nur unzureichend archiviert werden. Hierin liegt das größte Verdienst der Studie: einen ersten empirisch-chronologischen Überblick über Wahlspots im deutschen Fernsehen zu liefern.

Zudem macht die Autorin auf einige interessante nicht veröffentlichte Studien aufmerksam, wie beispielsweise Rüdiger Schmitt-Becks Untersuchung der „Wirkungen der Parteienwerbung im Fernsehen“, die im Rahmen einer Anhörung des Landtages Brandenburg Anfang 1999 erstellt wurde. Schmitt-Beck kommt mit Hinblick auf die Wirkung von Wahlspots rechtsradikaler Parteien zu dem vorläufigen Ergebnis, dass die Wirkung im Sinn von Wahlerfolg weniger auf die Spots selbst, als auf die durch Wahlspots ausgelösten Debatten und mittelbar auf die dadurch erzeugte Aufmerksamkeit der Nachrichtenmedien zurückzuführen ist: „Auf diese Weise gewinnen solche Parteien öffentliche Sichtbarkeit als wahlpolitische Alternativangebote für Wählerpotentiale, die schon vorher mit den von ihnen propagierten Auffassungen übereinstimmen" (SchmittBeck 1999: 3).

Holtz-Bacha betont die Bedeutung der visuellen Ebene der Fernsehspots und weist auf einen „Trend zum Spot ohne gesprochenen Text" (S. 235) hin, der allein auf die Wirkungskraft der Bilder setze. Dabei hätte sich gezeigt, "wie sehr sich politische Werbung und Wirtschaftswerbung gleichen" (S. 236). In Kapitel 5.6 der Langzeitstudie werden „Die Bilder der Spots“ gesondert untersucht. Zur Bildanalyse verwendet die Autorin jedoch keine spezifisch für die visuelle Analyse geeignete Methode, sondern konzentriert sich auf einen aus der Textinhaltsanalyse bekannten Ansatz des Vergleichs von Text- und Bildaussage einerseits sowie der Stereotypenanalyse andererseits. Die spezifische Eigenart visueller Kommunikation, die in einer ihr zugrundeliegenden assoziativen Logik besteht, im Unterschied zu textueller Kommunikation, die sich durch argumentative Strukturen auszeichnet, wird dadurch verkannt. Das Forschungsergebnis der Angleichung von politischer und kommerzieller Werbung könnte so auch als self-fulfilling prophecy gedeutet werden, die vor allem der gewählten Methode geschuldet ist. Denn eine eigene Motiv- und Bedeutungsanalyse der Bildsequenzen wird nicht vorgenommen. Vielmehr wurden die Bildeinstellungen nach bestimmten Werbestereotypen im Sinne von "Junge Leute-Typen“, „Sonntagstypen“, „Erfolgstypen“ und nach der Sichtbarkeit von Staatssymbolen wie etwa Parlamentsgebäuden oder Nationalflaggen kodiert. Die Gesamtaussage des Spots, der narrative Faden zwischen den Einstellungen und damit die eigentlichen Bedeutungsebenen können so nicht ermittelt werden.

Das im ersten Kapitel überzeugend beschriebene Forschungsziel, Wahlwerbung in einen erweiterten politikwissenschaftlichen Begriff der politischen Kultur als Deutungskultur einzubetten, wurde nur bedingt erreicht, da die gewählte Untersuchungsmethode die Bedeutungsebene und damit die Ebene der mentalen Vorstellungen nicht erschlossen hat. Dennoch ist die empirische Langzeitstudie einzigartig in der kommunikations- und politikwissenschaftlichen Forschungslandschaft und gehört in jede Fachbibliothek.

Marion G. Müller 